\title{
Endobronchial ultrasound in therapeutic bronchoscopy
}

\author{
F. Herth*, H.D. Becker*, J. LoCicero III Jr", A. Ernst ${ }^{\Uparrow}$
}

\begin{abstract}
Endobronchial ultrasound in therapeutic bronchoscopy. F. Herth, H.D. Becker, J. LoCicero III Jr, A. Ernst. (C) ERS Journals Ltd 2002.

ABSTRACT: Endobronchial ultrasound (EBUS) has been introduced as an adjunct to diagnostic bronchoscopy as it allows evaluation of the submucosal and parabronchial structures. Its use in therapeutic bronchoscopy has not been assessed. A large observational study of the value of EBUS in therapeutic bronchoscopy is presented here.

From January 1998-January 2001 all patients undergoing therapeutic bronchoscopy and EBUS were evaluated prospectively. Patient demographics, indication for bronchoscopy, interventional treatments used and changes in therapy as influenced by the use of EBUS were documented. A total 2,446 therapeutic bronchoscopies were performed. In 1,174 cases EBUS was used (29\% mechanical tumour debridement, $20 \%$ airway stenting, 13\% Neodymium:yttrium aluminium garnet (Nd:YAG) laser use, 23\% argon plasma coagulation, $11 \%$ brachytherapy, $2 \%$ foreign body removal and $2 \%$ endoscopic abscess drainage).

EBUS guided or changed therapy significantly in $43 \%$ of cases. Changes included adjustment of stent dimensions, termination of tumour debridement when nearing vessels, and referral for surgical interventions rather than endoscopic treatment. Complications associated with EBUS use were minimal. No patient undergoing EBUS guided tumour destruction experienced severe bleeding or fistula formation.

In summary, endobronchial ultrasound was easily performed and changed or guided therapeutic decisions during therapeutic bronchoscopic procedures in a substantial number of cases. As this may result in better outcomes, it has become a standard adjunct in the authors practice.
\end{abstract}

Eur Respir J 2002; 20: 118-121.

\author{
*Dept of Interdisciplinary Endoscopy, \\ Thoraxklinik-Heidelberg, Heidelberg, \\ Germany, ${ }^{\#}$ Thoracic Surgery and "Inter- \\ ventional Pulmonology, Beth Israel \\ Deaconess Medical Centre, Harvard \\ Medical School, Boston, MA, USA. \\ Correspondence: F. Herth \\ Thoraxklinik \\ Amalienstr. 5 \\ D-69126 Heidelberg \\ Germany \\ Fax: 496221396246 \\ E-mail: f@herth.net \\ Keywords: Airway obstruction \\ endobronchial ultrasound \\ interventional bronchoscopy \\ laser \\ stenting
}

Received: September 152001 Accepted after revision: January 16 2002
Therapeutic bronchoscopy is coming of age. Numerous procedures such as laser resection, argonplasma coagulation (APC), electrosurgery, cryotherapy, brachytherapy, photodynamic therapy (PDT) and stenting are now available to the endoscopist [1]. With all these options, sometimes there is a question of what intervention is best to use on an individual patient.

Many abnormalities leading to the need for interventional bronchoscopy arise from the airway wall and parabronchial structures [2]. There is a need to expand the view of the endoscopist beyond the confinement of the mucosal wall [3], especially in bronchoscopic treatment of malignant tumours with curative intent such as PDT and brachytherapy with endoluminal high-dose radiation (HDR). In these lesions, it is important to ensure that tumour growth is restricted to the bronchial wall [4] in order to achieve a good and lasting result.

There is evidence that endobronchial ultrasound (EBUS) is superior to conventional computed tomography scan of the lung in evaluating the tracheal and bronchial wall structures [5]. It could serve as an ideal tool for evaluating airway wall anatomy and tumour pathology related to it [3,6]. Even though endoscopic ultrasound has been in use for quite some time, especially in gastroenterology, it has been slower finding its way into bronchoscopy. This may in part, be due to the technical problems associated with ultrasound in air-filled structures [3, 4, 7], but also certainly because of the need for specialised education of endoscopists in interpreting the ultrasound images.

As commercial ultrasound devices are now available, the authors experience with endobronchial ultrasound is presented here and also an assessment of where its use may be a beneficial and a unique adjunct to therapeutic bronchoscopy.

\section{Patients and methods}

Between January 1998-January 2001 all patients undergoing therapeutic bronchoscopy and EBUS were documented prospectively. Bronchoscopy was performed by experienced pulmonologists in standard fashion either with the rigid instrument (most commonly combined with flexible instruments) under general anaesthesia and jet ventilation in a previously described fashion $[6,7]$ or with a flexible instrument under conscious sedation.

Endobronchial ultrasound was performed with a flexible $20 \mathrm{MHz}$ probe (UM-2R/3R with driving unit MH-240 and processor EU-M 20 and 30; Olympus, Tokyo, Japan) through the working channel of a flexible bronchoscope (models BF P20D, BF IT 1O, and BF IT 30; Olympus). Briefly, the probe was 


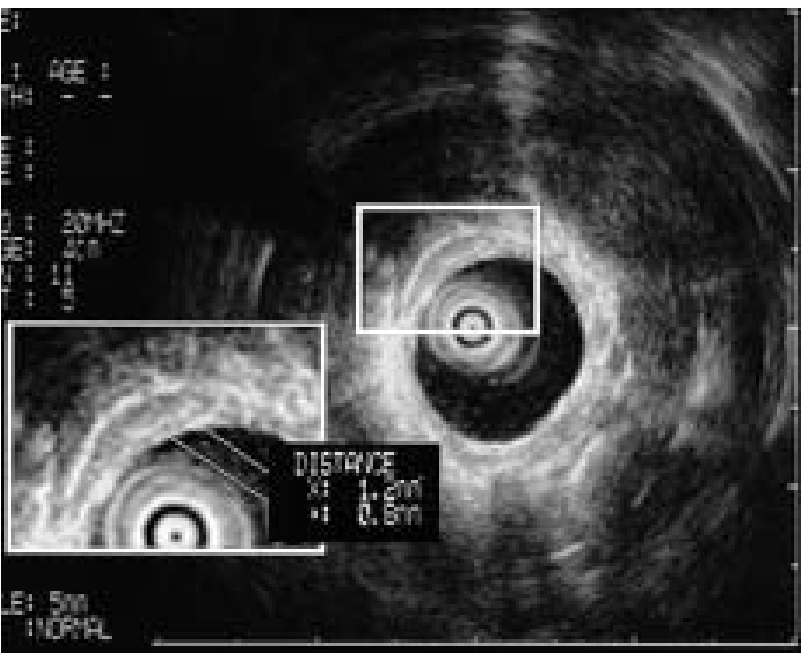

Fig. 1. - Example of the 360-degree image obtained by endobronchial ultrasound. A sector enlargement shows the ability to distinguish between different layers of the bronchial wall.

introduced into the airway and positioned in the desired location. As air causes reflection of the ultrasonic waves, a fluid-filled balloon at the tip of the probe was slowly inflated under endoscopic control, until coupling with the airway wall was achieved and a circular image was obtained on the screen. Under favourable conditions, a penetration depth of $5 \mathrm{~cm}$ can be achieved with this system (fig. 1). A detailed review of the technical aspect of the use of EBUS is available elsewhere $[2,4,5]$. Indications for therapeutic intervention, indication for EBUS and the results, especially if the findings resulted in change of therapy or therapy guidance, were recorded.

\section{Results}

Between January 1998-January $2001 \quad 10,236$ patients underwent bronchoscopy. Of those, 2,446 (24\%) were therapeutic (table 1). In 1,174 cases $(47 \%)$ EBUS was used; this constituted the reviewed population. Of the 1,174 patients, 360 were female and 814 were male, mean age of the group was 58.8 yrs (range $24-83$ yrs). The most common reasons for not performing EBUS in the remaining 1,272 patients were: known EBUS results from a prior study $(n=128)$, interventions in children $(n=134)$, emergent

Table 1. - Indications for therapeutic bronchoscopy in all 2,446 patients during the study period

\begin{tabular}{lc}
\hline Indication & $\mathrm{n}(\%)$ \\
\hline Mechanical tumour destruction & $723(30)$ \\
Stent placement & $489(20)$ \\
Nd:YAG laser resection & $289(12)$ \\
Argon-plasma coagulation & $426(17)$ \\
Brachytherapy & $296(12)$ \\
Foreign body removal & $156(6)$ \\
Abscess drainage & $67(3)$ \\
\hline
\end{tabular}

Nd:YAG: Neodymium:yttrium aluminium garnet.
Table 2. - Indications for therapeutic bronchoscopy in the study population with use of endobronchial ultrasound

\begin{tabular}{lc}
\hline Indication & $\mathrm{n}(\%)$ \\
\hline Mechanical tumour destruction & $346(29)$ \\
Stent placement & $235(20)$ \\
Nd:YAG laser resection & $148(13)$ \\
Argon-plasma coagulation & $262(23)$ \\
Brachytherapy & $134(11)$ \\
Foreign body removal & $22(2)$ \\
Abscess drainage & $27(2)$ \\
\hline
\end{tabular}

Nd:YAG: Neodymium:yttrium aluminium garnet. $n=1,174$.

interventions in life threatening situations $(n=421)$, bronchoscopy performed by an endoscopist untrained in EBUS interpretation $(\mathrm{n}=203)$ and technical problems $(n=189)$.

EBUS was used before mechanical tumour debridement (346 cases, 29\%), in airway stent placement (235 cases, 20\%), before and during Neodymium: yttrium aluminium garnet $(\mathrm{Nd}: \mathrm{YAG})$ laser use (148 cases, $13 \%$ ) and APC (262 cases, 23\%) for tumour destruction. EBUS was also used in the bronchoscopic treatment of malignant tumours with high-dose brachytherapy (HDR) in 134 cases $(11 \%)$. In patients, suffering from nonmalignant pulmonary disease with an indication for therapeutic bronchoscopy, EBUS was used in 22 cases $(2 \%)$ of foreign body removal and in endobronchial abscess drainage in 27 cases $(2 \%)$. As many patients were treated with more than one modality, only the treatments leading to the use of EBUS are listed in table 2. No patients were counted twice.

In $43 \%$ of the cases where EBUS was used, it resulted in change of therapy or better guidance of the intervention (table 3). Changes in treatment were, for example, the use of longer stents for endoscopically unappreciated submucosal or parabronchial tumour extension (fig. 2). Tumour debridement with laser or APC was stopped when EBUS demonstrated close relationships with vessels (fig. 3). Severe bleed or fistula formation did not develop in any of the cases where EBUS was used to guide tumour destruction.

When used for brachytherapy in patients with suspected early stage carcinoma, EBUS revealed local disease extension or lymph-node metastasis, which escaped all other imaging methods, in $28 \%$ of patients (fig. 4). Lymph-node metastasis was confirmed

Table 3.-Frequency in which planned therapeutic approaches were altered or guided by endobronchial ultrasound findings

\begin{tabular}{lr}
\hline Indication & $\mathrm{n}(\%)$ \\
\hline Mechanical tumour destruction & $123 / 346(36)$ \\
Stent placement & $121 / 235(51)$ \\
Nd:YAG laser resection & $56 / 148(38)$ \\
Argon-plasma coagulation & $121 / 262(46)$ \\
Brachytherapy & $69 / 134(51)$ \\
Foreign body removal & $3 / 22(14)$ \\
Abscess drainage & $12 / 27(42)$
\end{tabular}

Nd:YAG: Neodymium:yttrium aluminium garnet. 

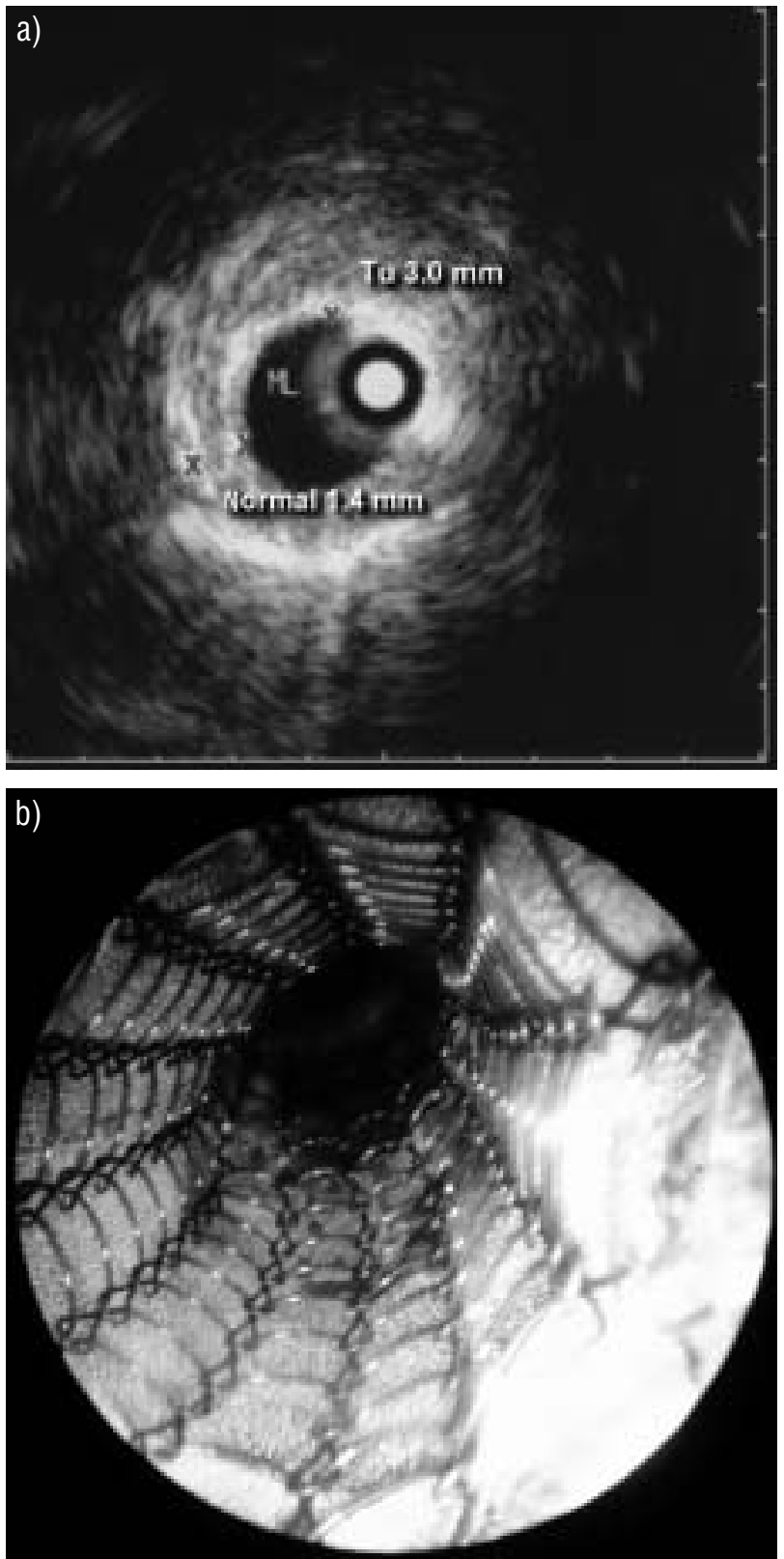

Fig. 2.-a) Endobronchial ultrasound image of a bronchus adjacent to an area with endobronchial obstruction. Submucosal tumour extension, which was not visible with conventional endoscopy, is documented. Normal thickness of the wall is compared to the infiltrated area. b) Endoscopic image of the stented airway (Ultraflex, Boston Scientific, Natick, MA, USA). A longer stent was used covering the airway portion involved with submucosal disease. Tu: tumour; X: border of the bronchial wall.

by EBUS guided transbronchial needle aspiration in all of these cases.

EBUS was used 293 times in the trachea (21\%), 319 times in the right main bronchus $(24 \%), 83$ times in the right upper lobe $(6 \%), 84$ times in the bronchus intermedius $(6 \%), 49$ times in the middle lobe $(4 \%), 41$ times in the right lower lobe (3\%), 217 times in the left main bronchus $(16 \%), 134$ times the left upper lobe $(10 \%), 57$ times in the left lower lobe $(4 \%)$ and 47 times in the peripheral lung $(4 \%)$. The mean total procedure time (including bronchoscopy and EBUS)

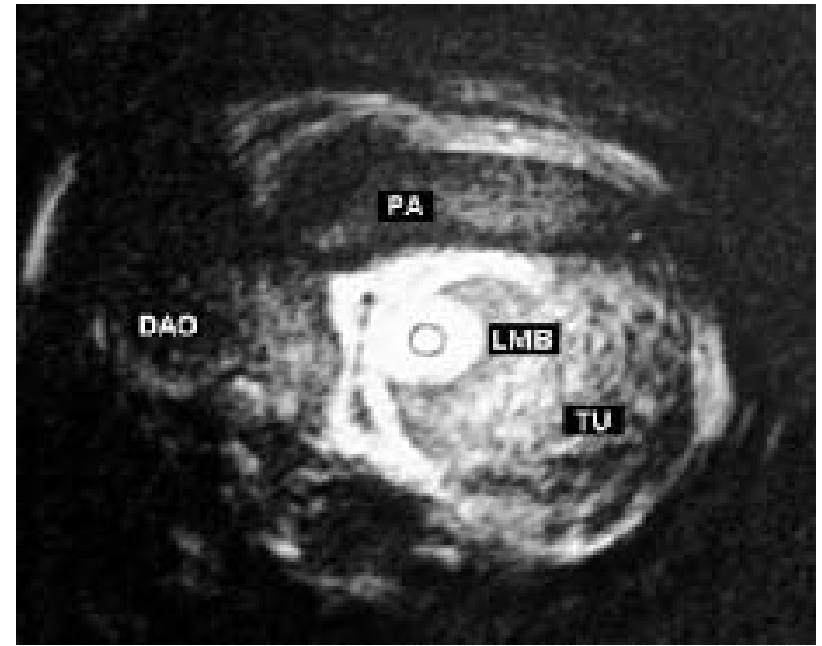

Fig. 3.-Endobronchial ultrasound image of a left mainstem bronchus (LMB) obstructed by malignant tumour (TU). The tumour is growing through the wall in very close proximity to the pulmonary artery (PA). Thermal tumour destruction can lead to the formation of a fistula and life threatening bleed. The descending aorta (DAO) is also shown.

was $23.2 \mathrm{~min}$ (range 5.7-43.5). The mean time for EBUS examinations was 5.3 min (range 3.1-14.4).

Cardiac arrhythmias, such as transient atrial tachycardia, were observed in 52 patients $(5.5 \%)$. This was associated with performing EBUS in the left lower lobe. All arrhythmias were self-limited and none caused haemodynamic compromise. No other complications associated with the use of EBUS were observed.

\section{Discussion}

Endobronchial ultrasound is a new addition to the diagnostic armamentarium of the bronchoscopist. Its properties allow for excellent visualisation of the bronchial wall and structures surrounding the airways and as such have significant potential to add to diagnostic bronchoscopies.

This is the first description of a large experience of the use of EBUS as a guide for therapeutic airway procedures. The addition of EBUS to conventional bronchoscopy changed the planned intervention in $43 \%$ of cases when it was used. The changes ranged from altering stent sizes to guiding tumour debridement. As EBUS affords a better assessment of the airway wall, longer stents were frequently chosen when submucosal infiltration was noted. This is not often appreciated by regular bronchoscopy.

A complication feared by interventionalists when using laser debridement in the airways is bleeding through neighbouring vessels or fistula formation. As EBUS allows for exact determination of tumour penetration through the airway wall and vessels are well visualized, debridement can be stopped when nearing critical structures. As a result, neither fistula formation or fatal bleeding was observed at all in this population.

When presented with a patient referred for curative 

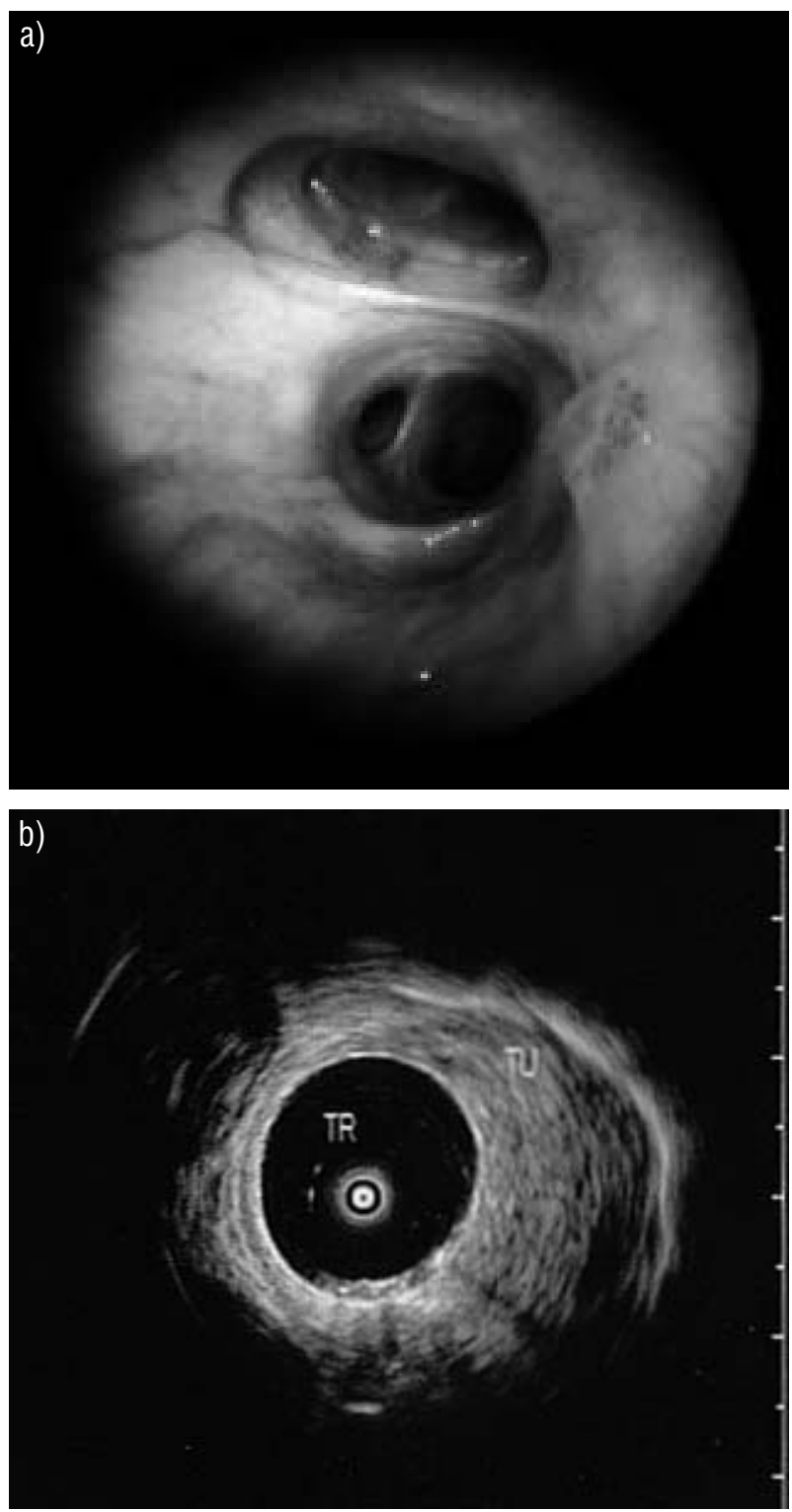

Fig. 4.-a) Endoscopic image of a small lesion in the airway, initially thought to be a carcinoma in situ and referred for photodynamic therapy (PDT). b) Endobronchial ultrasound of the airway demonstrating significant tumour extension into the submucosa with violation of the exterior wall. PDT would most likely not reach the entire tumour and surgical resection is the better option.

treatment of early airway malignancy, it is crucial to ascertain that local treatment has a high chance of success, as curative surgical resections should not be performed if the disease burden is too large for endoscopic treatment. In $28 \%$ of patients referred for presumed carcinoma in situ or early cancers EBUS established disease extent, which would have made endoscopic curative treatment impossible. EBUS may, as such, prove to be a good modality to stratify patients for surgical or endoscopic interventions of such lesions.

EBUS is simple to perform and not associated with any significant complications. In this large series only self-limited tachyarrhythmias when examining the left lower lobe were observed. None of the arrhythmias led to any haemodynamic compromises and none required therapy.

Obviously, not every therapeutic intervention requires the use of EBUS, as the information achieved may not be helpful. The authors currently use it in all cases of total airway obstruction before and during thermal tumour destruction as well as in all brachytherapy and PDT applications for planning purposes. In airway stenosis the authors use EBUS for malignant, but not for benign disease and in cases of abscess drainage or foreign body removal it is only used if there is a suspicion for airway abnormalities.

It is important to emphasise that this study is observational in nature and a significant number of patients who underwent therapeutic bronchoscopy did not have EBUS performed. The authors suspect that many of the cases where EBUS evaluation was not performed in this series would probably continue to be performed without EBUS, such as emergencies and previous assessments. Even though the authors feel that EBUS improves the safety of therapeutic procedures and can assist in decision making, it will be important to follow this encouraging report on a very large patient population with controlled-randomised trials to confirm the findings in specific patient populations.

For now endobronchial ultrasound has become a routine procedure in the authors' institutions and with the training of more pulmonologists in the use of this technology and image interpretation, endobronchial ultrasound will be a valuable addition to many bronchoscopic practises.

\section{References}

1. Turner JF, Ernst A, Becker HD. Rigid Bronchoscopy. J Bronchol 2000; 7: 171-176.

2. Freitag L. Tracheobronchial Stents. In: Bolliger CT, Mathur PN, eds. Progress in Respiratory Research Vol. 30. Interventional Bronchoscopy. Basel-Freiburg. S. Karger, 2000; pp. 171-185.

3. Becker HD, Herth F. Endobronchial ultrasound of the airways and the mediastinum. In: Bolliger CT, Mathur PN, eds. Progress in Respiratory Research Vol. 30. Interventional Bronchoscopy. Basel-Freiburg. S. Karger, 2000; pp. 80-93.

4. Sutedja G, Postmus PE. Bronchoscopic treatment of lung tumor. Lung Cancer 1994; 11: 1-17.

5. Herth F, Becker HD. Endobronchial ultrasound of the airways and the mediastinum. Monaldi Arch Chest Dis 2000; 55: 36-45.

6. Herth F, Becker HD. Endobronchial Ultrasound (EBUS) - assessment of a new diagnostic tool in bronchoscopy. Onkologie 2001; 24: 151-154.

7. Fischler M, Seigneur F, Bourreli B, Melchior JC, Lavaud C, Vourc'h G. Jet ventilation using low or high frequencies during bronchoscopy. $\mathrm{Br} J$ Anaesth 1985; 57: 382-388. 